\title{
Beschäftigungswirkungen arbeits- und sozialrechtlicher Schwellenwerte
}

\author{
Lena Koller · Claus Schnabel • Joachim Wagner
}

Angenommen: 25. Januar 2011 / Online publiziert: 10. Februar 2011

(C) Institut für Arbeitsmarkt- und Berufsforschung 2011

Zusammenfassung Im deutschen Arbeits- und Sozialrecht gibt es rund 160 Schwellenwerte. Diese geben an, $\mathrm{ab}$ welcher Beschäftigtenzahl ein bestimmtes Gesetz oder eine Regelung in einem Betrieb zur Anwendung kommt (z. B. Kündigungsschutzgesetz). Da das Überschreiten von Schwellenwerten meist mit zusätzlichen Kosten für die Betriebe verbunden ist (z. B. Gewährung von (Mitbestimmungs-)Rechten oder Einhaltung von Schutzstandards), wird vermutet, dass diese das Beschäftigungswachstum in Betrieben mit einer Beschäftigtenzahl unterhalb einer Schwelle bremsen. Umgekehrt wird Schwellenwerten unterstellt, dass sie den Beschäftigungsabbau in Betrieben oberhalb einer Schwelle fördern. Im Rahmen des DFG-Projekts wurde diesen Vermutungen nachgegangen. Dazu wurde auf Basis verschiedener Datensätze ökonometrisch überprüft, ob von der Beschäftigungspflicht für

Dieser Beitrag fasst die Ergebnisse unserer Arbeiten im Projekt „Betriebsgrößenabhängige Schwellenwerte und Arbeitsplatzdynamik in mittelständischen Firmen" zusammen, das Teil des DFG-Schwerpunktprogramms ,Flexibilisierungspotentiale bei heterogenen Arbeitsmärkten“" war. Wir danken der Deutschen Forschungsgemeinschaft für die finanzielle Förderung dieses Projekts.

L. Koller (凶)

Institut für Arbeitsmarkt- und Berufsforschung (IAB), Regensburger Str. 104, 90478 Nürnberg, Deutschland e-mail: lena.koller@iab.de

\section{Schnabel}

Lehrstuhl für Arbeitsmarkt- und Regionalpolitik, Friedrich-Alexander-Universität Erlangen-Nürnberg, Lange Gasse 20, 90403 Nürnberg, Deutschland

e-mail: claus.schnabel@wiso.uni-erlangen.de

\section{J. Wagner}

Institut für Volkswirtschaftslehre, Leuphana Universität Lüneburg, Postfach 2440, 21332 Lüneburg, Deutschland

e-mail: wagner@leuphana.de
Schwerbehinderte, der Freistellung des ersten Betriebsrats von seiner beruflichen Tätigkeit oder einer der 13 Regelungen rund um den 20er-Schwellenwert ein Einfluss auf die Beschäftigungsdynamik in Betrieben rund um die jeweilige Schwelle ausgeht, wobei sich nur selten die erwarteten Bremseffekte zeigten. Da Schwellenwertregelungen sehr unterschiedlich ausgestaltet sind, wurde zudem ein Vorschlag für deren Vereinheitlichung erarbeitet, um Transaktions- und Informationskosten zu senken und Ausweichreaktionen zu vermeiden.

Schlüsselwörter Schwellenwerte · Arbeitsnachfrage ·

Schwerbehinderte $\cdot$ Betriebsverfassungsgesetz .

Reformvorschlag

\section{JEL Klassifikationen J23}

\section{Employment effects of thresholds in labour law} Abstract In Germany, there exist around 160 threshold val-
ues stating that certain labour laws and regulations apply
only in establishments above a critical size (usually defined
by the number of employees). As crossing a threshold may
result in additional costs for the firm, it is often hypoth-
esized that firms try not to exceed thresholds, in such a
way dampening employment growth. Similarly, firms might
reduce employment in order to get rid of certain regula-
tions applying above a threshold. In our project, we have
tested these hypotheses using different data sets and in-
vestigating several labour laws (such as the Disabled Per-
sons Act, the Works Constitution Act, and various regu-
lations creating thresholds around the size of 20 employ-
ees in a firm). In contrast to expectations, very few effects
on employment dynamics have been found. Since thresh-
olds are defined inconsistently, we have developed several 
Anzahl überschrittener Schwellenwerte

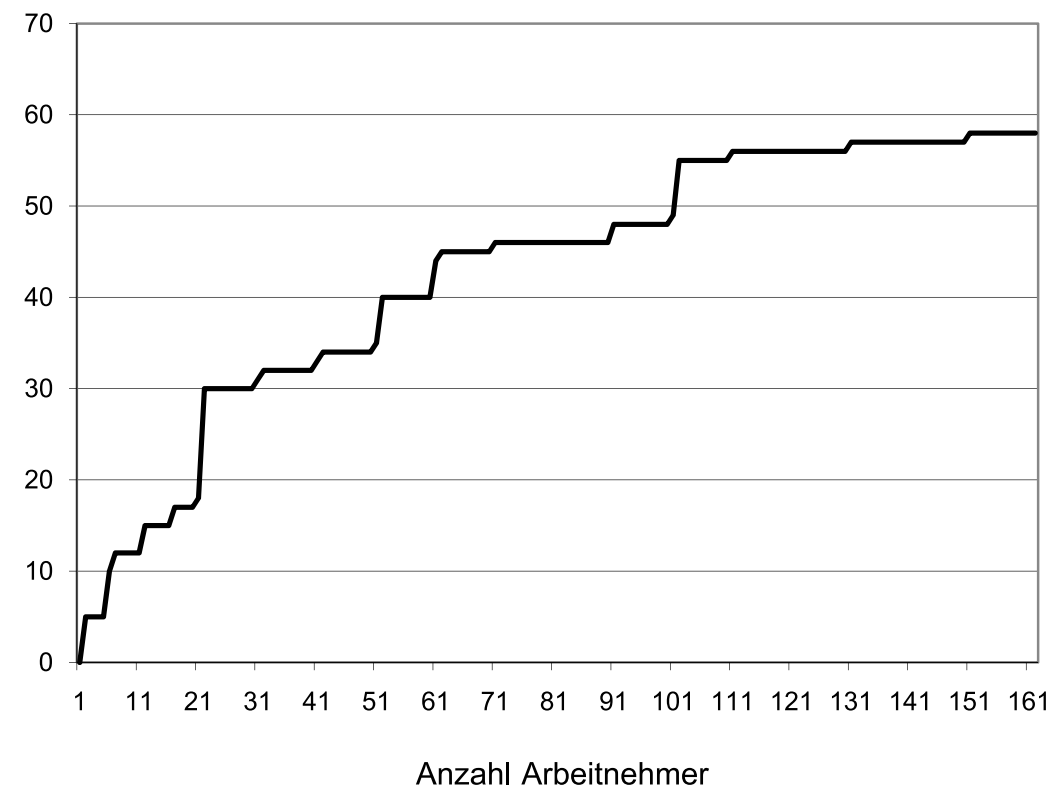

Hinweis: Die Zahl der Arbeitnehmer umfasst auch Arbeitsplätze und Besatzungsmitglieder auf Schiffen, unabhängig vom Bezugspunkt (Betrieb, Unternehmen, Konzern, Arbeitgeber, Arbeitsstätte oder Schiff). Schwellenwerte, die sich auf bestimmte Personengruppen (wie Jugendliche, Leitende Angestellte) beziehen, wurden nicht berücksichtigt. Als Schwellenwerte wurden auch die einzelnen Schwellen von Schwellenwertstaffeln gezählt.

Quelle: eigene Darstellung

Fig. 1 Anzahl der überschrittenen arbeitsrechtlichen Schwellenwerte nach Anzahl der Arbeitnehmer, kumuliert (Stand 2010)

suggestions for simplifying and unifying threshold regulations, which should reduce transaction costs and side effects.

\section{Einleitung}

Arbeits- und sozialrechtlichen Schwellenwerten wird häufig unterstellt, dass sie einen Einfluss auf die Arbeitsplatzdynamik in mittelständischen Firmen haben. Schwellenwerte geben an, ab welcher Beschäftigtenzahl ein bestimmtes Gesetz oder eine Regelung in einem Betrieb zur Anwendung kommt (z. B. Kündigungsschutzgesetz). Da die Umsetzung vieler arbeits- und sozialrechtlicher Regelungen mit zusätzlichen Kosten für die Unternehmen verbunden ist (z. B. Anwendung des Kündigungsschutzgesetzes oder Freistellung von Betriebsratsmitgliedern), wurden Schwellenwerte eingeführt, um kleine und mittlere Unternehmen von den Regelungen auszunehmen und dadurch von den Kosten zu befreien. Als Begründung wird von ökonomischer und arbeitsrechtlicher Seite meist die besondere Schutzbedürftigkeit kleinerer Unternehmen genannt. Überschreitet nun ein Betrieb mit seiner Beschäftigtenzahl einen Schwellenwert, so treten die Regelungen in Kraft und damit auch die mit ihnen verbunden Kosten. Daher wird vermutet, dass Betrie- be auf ein Beschäftigungswachstum über eine Schwelle verzichten, um die damit verbunden Kosten zu vermeiden. Umgekehrt könnten Betriebe ihre Beschäftigtenzahl unter einen Schwellenwert verringern, um dadurch zusätzliche Einsparungen zu erhalten.

In diesem Beitrag wird daher den Vermutungen nachgegangen, dass Schwellenwerte das Beschäftigungswachstum in Betrieben bremsen bzw. den Beschäftigungsabbau fördern. Das folgende Kapitel stellt zunächst die rechtstechnische Ausgestaltung arbeits- und sozialrechtlicher Schwellenwerte dar. Im Anschluss daran wird die relevante ökonomische Theorie erläutert. Abschnitt 4 fasst zuerst den Stand der Forschung zu Beginn des Projekts zusammen, bevor in Abschn. 5 die Ergebnisse zu den Beschäftigungswirkungen von Schwellenwerten, die im Rahmen des DFG-Projekts entstanden sind, erläutert werden. Der Beitrag schließt mit einem Vorschlag zur Vereinheitlichung der Schwellenwerteregelungen.

\section{Ausgestaltung von Schwellenwerten}

Derzeit gibt es in Deutschland rund 160 verschiedene Einzelschwellenwerte und Schwellenwertstaffeln. Während bei 
Einzelschwellenwerten nur ein Schwellenwert in der Regelung existiert (z. B. Geltungsbereich des Kündigungsschutzgesetzes in Betrieben mit mehr als 10 Arbeitnehmern ( $\$ 23$ KSchG)), nehmen bei Schwellenwertstaffeln die Vorschriften mit der Anzahl der Arbeitnehmer zu. Diese finden sich z. B. im Betriebsverfassungsgesetz. Dort steigt die Anzahl der Betriebsratsmitglieder, wenn die Anzahl der Arbeitnehmer im Betrieb mindestens 5, 21, 51, 101, 201 usw. beträgt ( $\$ 9$ BetrVG). In Abb. 1 sind die Schwellenwerte für den Bereich bis 161 Arbeitnehmer abgebildet. Sie zeigt, wie die Zahl der Regelungen durch das Überschreiten der Schwellen mit zunehmender Mitarbeiterzahl steigt.

Eine kleine Auswahl von Schwellenwertregelungen und deren rechtstechnische Ausgestaltung findet sich in Tab. 1. ${ }^{1}$ Sie verdeutlicht, dass sich Schwellenwerte in vielen Aspekten unterscheiden. So sind beispielsweise einige Schwellenwerte als „Mindest"-Werte konzipiert, andere dagegen als „mehr als"-Werte. Einige Regelungen beziehen sich auf die Anzahl der ,in der Regel“ Beschäftigten, andere auf die Anzahl der Arbeitnehmer zu einem bestimmten Zeitpunkt oder die ,jahresdurchschnittlich monatliche“ Anzahl an Arbeitsplätzen. Ferner beziehen sich manche Schwellenwerte auf die Arbeitnehmer eines Unternehmens, andere auf die eines Betriebes oder eines Arbeitgebers. Auch die Arbeitnehmer werden nicht in jeder Regelung gleich angerechnet. So werden etwa Teilzeitbeschäftigte in einigen Regelungen zeitanteilig gewichtet (,pro rata temporis“), in anderen aber wie Vollzeitkräfte gezählt. Daneben weisen die Regelungen auch Unterschiede hinsichtlich der Stellung der Arbeitnehmer im Beruf auf. Beispielsweise zählen Heimarbeiter, Leitende Angestellte oder Auszubildende nicht in allen Regelungen zu den Arbeitnehmern (vgl. Koller 2007).

Da Schwellenwerte jedoch nicht nach einem klaren, konsistenten System in die Gesetze eingeführt worden sind und rund die Hälfte der Regelungen seit 1993 zum Teil mehrfach geändert wurde (wie etwa die dreimalige Veränderung des Schwellenwerts im Kündigungsschutzgesetz seit 1996), ist im Laufe der Zeit ein ziemlicher „Wildwuchs“ von uneinheitlich gestalteten Schwellenwerten entstanden. Anstatt dass Schwellenwerte somit zu einer Entlastung bei den Betrieben führen, belasten sie diese durch erhöhte Transaktionskosten und Risiken. So ist es für Unternehmen nicht nur wegen der großen Zahl von rund 160 Schwellenwertregelungen, sondern auch wegen deren äußerst unterschiedlicher Ausgestaltung schwierig, den Überblick über alle Regelungen zu behalten.

\footnotetext{
${ }^{1}$ Für eine systematische Übersicht über alle existierenden Regelungen und deren Ausgestaltung zum Stand von November 2008 sei auf Koller (2010) verwiesen.
}

\section{Theoretische Überlegungen zu den Beschäftigungswirkungen von Schwellenwerten}

Im Folgenden wird erläutert, inwiefern Schwellenwerte das betriebliche Beschäftigungswachstum beeinflussen können. Existiert in einer Regelung ein Schwellenwert, so sind Unternehmen, deren Arbeitnehmerzahl unter dieser Schwelle liegt, von der betreffenden Regelung befreit. Wird der Schwellenwert jedoch überschritten, kommt die Regelung zur Anwendung, was meist mit Verpflichtungen für die Unternehmen verbunden ist. Diese Verpflichtungen bestehen oft in der Gewährung von (Mitbestimmungs-)Rechten für die Arbeitnehmer oder der Einhaltung bestimmter Schutzstandards, welche für die Sicherheit der Arbeitnehmer Sorge tragen. Gleichzeitig verursachen derartige Verpflichtungen häufig zusätzliche Kosten bei den Unternehmen. Diese bestehen beispielsweise in den Kosten der Betriebsratstätigkeit oder der Umsetzung von Maßnahmen zur Arbeitssicherheit (vgl. Koller et al. 2007).

Befindet sich nun ein Unternehmen mit seiner Beschäftigtenzahl direkt vor einer Schwelle und steigt die Nachfrage nach den vom ihm produzierten Gütern, so können dem Unternehmen, falls es seine Produktion ausweitet und dies mit einem Beschäftigungswachstum über die Schwelle verbunden ist, durch das Inkrafttreten der jeweiligen Regelung zusätzliche Kosten entstehen. Daher wird vermutet, dass insbesondere Schwellenwerte, deren Überschreiten mit hohen Kosten für die Unternehmen verbunden ist, die Schaffung zusätzlicher Arbeitsplätze in Unternehmen vor einer Schwelle bremsen. Umgekehrt kann bei einem Nachfragerückgang mit entsprechendem Beschäftigungsabbau ein Unterschreiten des Schwellenwerts durch den Wegfall der Regelungen zu zusätzlichen Kosteneinsparungen führen. Dies impliziert, dass Schwellenwerte daher in Unternehmen direkt über einer Schwelle einen Anreiz für Entlassungen darstellen (vgl. Koller 2010).

Staatliche Sozialpolitik könnte sich somit negativ auf die gesamtwirtschaftliche Wohlfahrt auswirken, wenn Unternehmen aufgrund der Schwellenwerte die gestiegene Nachfrage nach Gütern nicht befriedigen, sondern auf ein Beschäftigungswachstum und damit eine Produktionssteigerung verzichten, um die zusätzlichen Kosten zu vermeiden. Ferner könnten Wohlfahrtsverluste entstehen, wenn Unternehmen ihre Beschäftigung abbauen, um unter eine Schwelle zu gelangen und zusätzliche Kosten zu sparen.

\section{Stand der Forschung}

Der Vermutung, Schwellenwerte könnten einen Einfluss auf die Beschäftigungsdynamik in Betrieben haben, wurde bereits in verschiedenen Betriebsbefragungen und ökonometrischen Analysen nachgegangen. Allerdings lagen zu Be- 


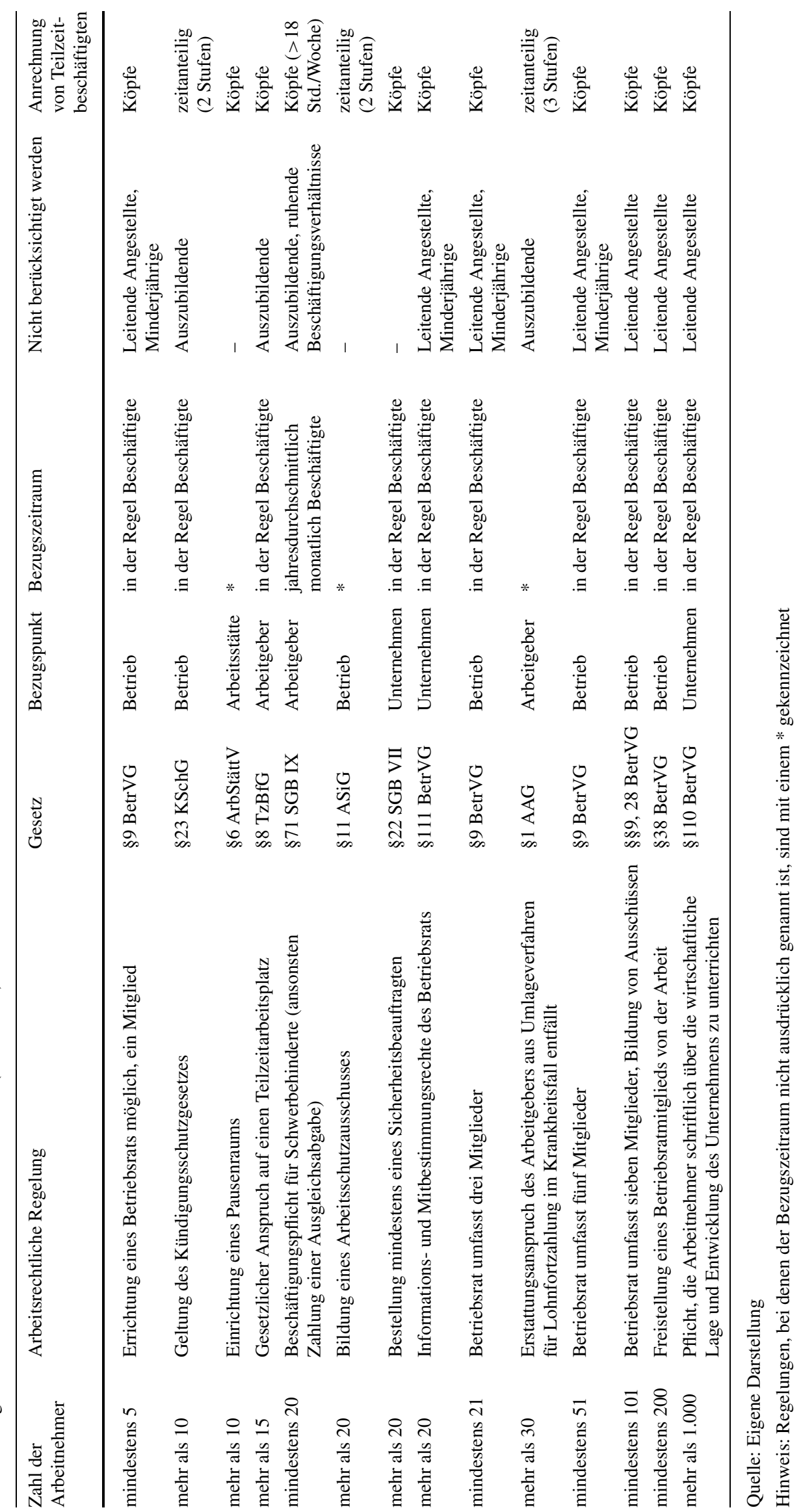


ginn des DFG-Projekts - mit einer Ausnahme - nur Ergebnisse von Studien vor, die sich mit den Beschäftigungswirkungen des Schwellenwerts, der den Geltungsbereich des Kündigungsschutzgesetzes ( $\$ 23 \mathrm{KSchG}$ ) angibt, befasst haben. $^{2}$

Eine Befragung, welche sich mit den Beschäftigungswirkungen von Schwellenwerten im Allgemeinen befasste, wurde Friedrich und Hägele (1997) in rund 1.500 Unternehmen durchgeführt. Sie stellten fest, dass 19 Prozent der befragten Unternehmen Einstellungen so lange wie möglich hinauszögerten, um Schwellenwerte nicht zu überschreiten. Weitere 22 Prozent der Unternehmen gaben an, konsequent unter bestimmten Schwellenwerten zu bleiben. Eine Umfrage des Deutschen Industrie- und Handelstags (1998) unter rund 300 Unternehmen mit fünf bis zehn Arbeitnehmern ergab, dass 11 Prozent der Unternehmen seit der Erhöhung des Schwellenwerts im Oktober 1996 von ,,mehr als fünf“ auf „mehr als zehn“ Arbeitnehmern Personal eingestellt hatten. Weitere 14 Prozent wollten deswegen in $\mathrm{Zu}$ kunft noch einstellen. FORSA kam ebenfalls zu dem Ergebnis, dass 14 Prozent der rund 1.000 befragten Betriebe mit bis zu fünf Beschäftigten in den fünf Jahren seit der neuerlichen Herabsetzung des Schwellenwertes 1999 auf „,mehr als fünf“ Arbeitnehmer auf die Einrichtung zusätzlicher Arbeitsplätze verzichtet hatten (Institut der deutschen Wirtschaft Köln 2003). Von den Betrieben mit genau fünf Beschäftigten gaben sogar 31 Prozent der Betriebe an, auf Einstellungen verzichtet zu haben, um nicht in den Geltungsbereich des Kündigungsschutzgesetzes zu gelangen.

Die erste ökonometrische Analyse, die sich mit den Beschäftigungseffekten des Schwellenwerts im Kündigungsschutzgesetz befasste, wurde von Wagner et al. (2001) auf Basis des IAB-Betriebspanels für die Jahre 1993 bis 1996 durchgeführt. Sie kamen anhand von Schätzungen eines Random-Effects-Probit-Modells zu dem Ergebnis, dass sich die Wahrscheinlichkeit einer Erhöhung der Beschäftigtenzahl in Betrieben vor der Schwelle, die einen Anstieg des Geschäftsvolumens erwartet hatten, nicht signifikant von der anderer vergleichbarer Betriebe unterscheidet. Verick (2004) führte - unter Verwendung des IAB-Betriebspanels - Difference-in-Difference-Probit-Schätzungen zur Wahrscheinlichkeit einer Beschäftigungsveränderung durch. Seine Analysen ergaben zwar, dass die Wahrscheinlichkeit, die Beschäftigung unverändert zu lassen, nach der Verringerung des Schwellenwerts 1999 (von „,mehr als 10“

\footnotetext{
${ }^{2}$ Daneben gibt es noch weitere Studien, die den Schwellenwert im Kündigungsschutzgesetz berücksichtigen. Diese untersuchen jedoch entweder die Beschäftigung befristet Beschäftigter (siehe hierzu Hagen und Bookmann 2002 sowie Fritsch und Schank 2005) oder die Arbeitsplatzdynamik (siehe Bauer et al. 2007) in Betrieben rund um den Schwellenwert und nicht dessen Einfluss auf das Beschäftigungswachstum.
}

auf „mehr als 5“ Arbeitnehmer) in Betrieben mit maximal fünf Arbeitnehmern signifikant höher war als in Betrieben mit sechs bis 13 Arbeitnehmern. Robustheitschecks konnten dieses Ergebnis jedoch nicht bestätigen. Auch Burgert (2006) fand - mit Hilfe des IAB-Betriebspanels von 1996 bis 2001 - unter Anwendung eines Regression Discontinuity Designs keine Hinweise darauf, dass die Veränderungen des Schwellenwerts 1996 und 1999 das Beschäftigungswachstum von Betrieben unter dem Schwellenwert im Vergleich zu Betrieben oberhalb signifikant verringert haben.

Während nun Befragungen insbesondere dem Schwellenwert, der den Geltungsbereich der Kündigungsschutzgesetzes bestimmt, einen bremsenden Einfluss auf das Beschäftigungswachstum unterstellen, konnte keine der ökonometrischen Analysen einen eindeutig bremsenden Effekt dieses Schwellenwerts nachweisen.

\section{Ergebnisse der ökonometrischen Analysen im Rahmen des DFG-Projekts}

Wie im vorangegangenen Kapitel erläutert, lagen zu Beginn des DFG-Projekts „Betriebsgrößenabhängige Schwellenwerte und Arbeitsplatzdynamik in mittelständischen Firmen" vor allem Erkenntnisse über die Beschäftigungswirkungen eines Schwellenwerts vor. Ziel des Projekts war es daher, die Beschäftigungswirkungen weiterer (kostenintensiver) Schwellen zu untersuchen. Im Fokus standen dabei nicht nur die Auswirkungen der Schwellenwerte auf das Beschäftigungswachstum, sondern auch die Effekte auf den Beschäftigungsabbau. Analysiert wurde, ob die Beschäftigungspflicht für Schwerbehinderte, die Freistellung des ersten Betriebsrats von seiner beruflichen Tätigkeit sowie Regelungen mit einem Schwellenwert rund um den 20erSchwellenwert einen Einfluss auf die Beschäftigungsdynamik haben.

In einem ersten Schritt wurden die Effekte der Beschäftigungspflicht für Schwerbehinderte ökonometrisch analysiert. Dazu wurde von Koller et al. (2006) auf Basis der St 88 (,Statistik aus dem Anzeigeverfahren gem. §77 Abs. 2 SGB IX“) und dem IAB-Betriebspanel geprüft, ob die Pflicht, ab 24 Arbeitsplätzen mindestens zwei Schwerbehinderte zu beschäftigten oder andernfalls eine Ausgleichsabgabe zu zahlen, einen bremsenden Effekt auf die Wahrscheinlichkeit eines Beschäftigungswachstums hatte. Überprüft wurde auBerdem, ob die Beschäftigungspflicht die Wahrscheinlichkeit eines Beschäftigungsabbaus gefördert hat. Ihre Probitanalysen ergaben, dass Arbeitgeber, die 24 Arbeitsplätze im Jahr 1999 und nicht ausreichend Schwerbehinderte beschäftigt hatten (und deshalb bei Überschreiten des Schwellenwerts eine Ausgleichsabgabe zahlen oder einen Schwerbehinderten einstellen mussten), mit einer signifikant geringeren Wahrscheinlichkeit im Jahr 2000 die Schwelle 
zur Beschäftigungspflicht eines weiteren Schwerbehinderten überschritten haben als andere Arbeitgeber. Berechnungen zur Größenordnung des Bremseffekts beim Beschäftigungsaufbau ergaben, dass auf Grund der zweiten Schwelle des Schwerbehindertengesetzes maximal 2.500 Arbeitsplätze nicht eingerichtet worden sind. Die Probitschätzungen zum Einfluss des Schwellenwerts auf den Beschäftigungsabbau ergaben dagegen, dass Arbeitgeber, die sich 1999 mit 25 Arbeitsplätzen direkt über der Schwelle befanden und eine Ausgleichsabgabe zahlen mussten, keine signifikant höhere Wahrscheinlichkeit hatten, ihre Beschäftigtenzahl zu verringern, als andere Arbeitgeber.

Über 60 der rund 160 Schwellenwerte finden sich im Betriebsverfassungsgesetz. Daher lag es nahe, im Rahmen des Projekts die Beschäftigungswirkungen des Schwellenwerts zur Freistellung des ersten Betriebsratsmitglieds von der beruflichen Tätigkeit zu untersuchen. Koller et al. (2008) verwendeten dazu Angaben aus dem Betriebs-Historik-Panel der Bundesagentur für Arbeit (BHP). Sie analysierten dabei nicht nur die Wirkungen der Freistellung des ersten Mitglieds in Betrieben ab 200 Beschäftigten, sondern nutzen auch die Herabsetzung dieses Schwellenwerts im Juli 2001 von 300 auf 200 Arbeitnehmern als natürliches Experiment. Mittels Probitanalysen haben Koller, Schnabel und Wagner untersucht, ob der Schwellenwert von 300 Arbeitnehmern im Zeitraum von Juni 1999 bis Juni 2001 bzw. der Schwellenwert von 200 Arbeitnehmern im Zeitraum von Juni 2001 bis Juni 2003 eine bremsende Wirkung auf die Wahrscheinlichkeit eines Beschäftigungswachstums für Betriebe hatte. Zudem konnten sie an Hand eines Differenzvon-Differenzen-Ansatzes überprüfen, ob die Herabsetzung des Schwellenwerts das Beschäftigungswachstum von Betrieben beeinflusst hat. Die Ergebnisse zeigen, dass weder der alte Schwellenwert von 300 Arbeitnehmern noch der neue Schwellenwert von 200 Arbeitnehmern oder dessen Änderung eine bremsende Wirkung auf das Beschäftigungswachstum von Betrieben hatte. Weitere Analysen zu den Auswirkungen der Freistellung auf die Wahrscheinlichkeit eines Beschäftigungsabbaus von Koller (2010) führten zu gleichen Ergebnissen: Weder der alte noch der neue Schwellenwert oder dessen Änderung Herabsetzung hatten einen signifikanten Effekt auf den Beschäftigungsabbau in Betrieben.

Wie die Abb. 1 zeigt, müssen insbesondere Kleinbetriebe viele Schwellenwertregelungen beachten. So liegen beispielsweise Betriebe mit 25 Arbeitnehmern mit ihrer Beschäftigtenzahl über 30 Schwellenwerten. Daher wurde von Koller (2010) auf Basis des BHP untersucht, ob es Auffälligkeiten im Wachstumsverhalten von Kleinbetrieben mit weniger als 30 Arbeitnehmern gibt. Für die Analysen der Beschäftigungswirkungen von Schwellenwerten auf Kleinbetriebe wurde die Beschäftigtenzahl der Betriebe jeweils entsprechend der gesetzlichen Bestimmungen berechnet (dabei wurde u. a. berücksichtigt, ob Auszubildende zur schwellenrelevanten Beschäftigtenzahl zählen und wie die Teilzeitbeschäftigte gewichtet werden). Insgesamt wurde die Beschäftigtenzahl auf sechs verschiedene Arten berechnet. Die deskriptiven Analysen ergaben, dass es zwischen 1993 und 2005 keine Auffälligkeiten beim Anteil an Betrieben mit einer bestimmten Beschäftigungsveränderung gab, die mit einem Schwellenwert korrespondiert hätte. Auch die Verwendung von Übergangsmatrizen sowie die ökonometrische Überprüfung mittels OLS-Regressionen und semiparametrischen Schätzverfahren zeigte keine Besonderheiten in Bezug auf die Betriebsgröße. Dies gilt auch für den Schwellenwert zum Inkrafttreten des Kündigungsschutzgesetzes. Keinem der jeweils gültigen Schwellenwerte konnte unter Berücksichtigung der jeweils geltenden gesetzlichen Bestimmungen zur Berechnung der Beschäftigtenzahl ein Beschäftigungseffekt nachgewiesen werden.

Von Koller (2010) wurde ferner analysiert, ob von einer der 13 Regelungen mit einem Bezug zu 20 Arbeitnehmern Auswirkungen auf die Beschäftigungsveränderung von 2004 auf 2005 ausgegangen sind. Datenbasis war wieder das BHP, und die Beschäftigtenzahl wurde entsprechend der gesetzlichen Vorgaben bestimmt. Die Ergebnisse zeigen, dass die Wahrscheinlichkeit eines Beschäftigungsaufbaus in Betrieben mit einer Beschäftigtenzahl knapp unterhalb der jeweiligen Schwelle im Vergleich zu anderen Betrieben nicht signifikant geringer ist. Auch von der Ballung der Schwellenwerte um die 20er-Schwelle scheinen keine Beschäftigungseffekte auszugehen. So hatten Betriebe, die 2004 mit 20 vollzeitbeschäftigten und volljährigen Arbeitnehmern - unabhängig von der jeweiligen Berechnungsmethode der Beschäftigtenzahl - immer direkt unterhalb der zwölf Schwellenwerte von „mehr als 20 Arbeitnehmern“ lagen und bei denen bei einem Beschäftigungswachstum alle zwölf Regelungen gleichzeitig zur Anwendung kamen, keine signifikant geringere Wahrscheinlichkeit eines Beschäftigungswachstums als andere Betriebe. Auch in Bezug auf die Wahrscheinlichkeit einer Beschäftigungsveränderung wurden die bisherigen Ergebnisse bestätigt. So war weder die Wahrscheinlichkeit eines Beschäftigungsabbaus in Betrieben knapp über einer bestimmten Schwelle höher als bei anderen Betrieben, noch verringerten Betriebe, die 2004 mit 21 vollzeitbeschäftigten und volljährigen Arbeitnehmern über allen zwölf Schwellen lagen, ihre Beschäftigung signifikant häufiger. Die Annahme, dass die Ballung von Schwellenwerten den Bremseffekt verstärkt bzw. den Anreiz eines Beschäftigungsabbaus erhöht, konnte somit nicht bestätigt werden.

Zusammenfassend lässt sich festhalten, dass weder einzelne Schwellenwerte noch die Ansammlung von Schwellenwerten bei einer bestimmten Beschäftigtenzahl das Beschäftigungswachstum in Betrieben unterhalb dieser Schwellen bremst. Umgekehrt konnte nicht belegt werden, 
dass Schwellenwerte den Beschäftigungsabbau in Betrieben über diesen Schwellen fördern. Eine Ausnahme stellt nur der Schwellenwert zur Beschäftigungspflicht von zwei Schwerbehinderten dar, dem ein signifikant bremsender Effekt auf das Beschäftigungswachstum in Unternehmen unterhalb der Schwelle nachgewiesen werden konnte. Allerdings ist die ökonomische Bedeutung dieses Bremseffekts als eher gering einzuschätzen.

\section{Wirtschaftspolitische Empfehlungen}

Wie die Analysen gezeigt haben, gehen von beschäftigtenzahlabhängigen Schwellenwerten im deutschen Arbeitsund Sozialrecht keine substanziellen Effekte auf die Arbeitsplatzdynamik in mittelständischen Firmen aus. Die Schwellenwertproblematik scheint somit weniger ausgeprägt zu sein als oftmals von Praktikern behauptet. Allerdings ist es für Unternehmen mit hohem personellen oder finanziellen Aufwand und Ineffizienzen verbunden, den Überblick über die sie betreffenden Schwellenwerte zu erlangen und zu behalten, weil von den rund 160 verschiedenen arbeits- und sozialrechtlichen Schwellenwerten nur wenige auf die gleiche Art berechnet werden.

Da eine Reduzierung und einheitlichere Ausgestaltung von Schwellenwerten Transaktionskosten senken und die ökonomische Effizienz erhöhen könnte, wurde bereits ein Reformvorschlag zur Vereinheitlichung der Berechnung von Schwellenwerten erarbeitet (vgl. Koller et al. 2007 sowie Schnabel 2007). Dieser sieht vor, dass alle Schwellenwerte einheitlich in Form von „Mindest“-Werten ausgedrückt werden und der Eingangsschwellenwert für die Anwendung von Arbeits- und sozialrechtlichen Regelungen auf „mehr als 20 Arbeitnehmer" festgesetzt wird. Sollte eine solche einheitliche Regelung auf relativ hohem Niveau politisch nicht durchsetzbar sein, kann ersatzweise die Schwelle von „mehr als zehn Arbeitnehmern" Verwendung finden. In Kraft treten sollen die Regelungen erst, wenn der Schwellenwert an drei aufeinanderfolgenden Stichtagen, die jeweils in der Mitte eines Quartals liegen, überschritten wird. Bezugspunkt soll in der Regel der Betrieb sein, da dieser üblicherweise die arbeitsorganisatorische Einheit für rechtliche Regelungen zum Schutz des Arbeitnehmers in seiner konkreten Arbeitssituation darstellt. Teilzeitbeschäftigte sollen einheitlich in zwei Stufen (Gewichtung der Teilzeitbeschäftigten mit weniger als 20 Stunden pro Woche mit einem Faktor von 0,5 und Teilzeitbeschäftigte mit 20 bis unter 30 Stunden pro Woche mit einem Faktor von 0,75) gewichtet werden. Dies verhindert eine überproportionale Belastung von Betrieben mit vielen Teilzeitbeschäftigten und wirkt der Aufspaltung von Arbeitsplätzen entgegen. Auszubildende, Leiharbeitnehmer und Arbeitnehmer in ruhenden Beschäftigungsverhältnissen sollen bei der Zahl der Arbeitnehmer nicht berücksichtigt werden.
Zwar wäre eine derartige Vereinheitlichung und Vereinfachung der Schwellenwertregelungen mit einer Vielzahl von Gesetzesänderungen verbunden und die Kosten für die Unternehmen würden zunächst steigen, da sie sich mit den neuen Regelungen vertraut machen müssen. In Zukunft dürfte jedoch die Überschaubarkeit erhöht und Informations- und Bürokratiekosten könnten für die Unternehmen gesenkt werden. Schwellenwerte hätten somit wieder ihre eigentliche Funktion und könnten kleine und mittlere Unternehmen vor übermäßigen Belastungen durch das Arbeits- und Sozialrecht befreien.

\section{Kurzfassung}

Arbeits- und sozialrechtlichen Schwellenwerten wird häufig unterstellt, dass sie einen Einfluss auf die Arbeitsplatzdynamik in mittelständischen Firmen haben. Schwellenwerte geben an, ab welcher Beschäftigtenzahl ein bestimmtes Gesetz oder eine Regelung in einem Betrieb zur Anwendung kommt (z. B. Kündigungsschutzgesetz). Da die Umsetzung vieler arbeits- und sozialrechtlicher Regelungen mit zusätzlichen Kosten für die Unternehmen verbunden ist (z. B. Anwendung des Kündigungsschutzgesetzes oder Freistellung von Betriebsratsmitgliedern), wurden Schwellenwerte eingeführt, um kleine und mittlere Unternehmen von den Regelungen auszunehmen und dadurch von den Kosten zu befreien. Als Begründung wird von ökonomischer und arbeitsrechtlicher Seite meist die besondere Schutzbedürftigkeit kleinerer Unternehmen genannt. Überschreitet nun ein Betrieb mit seiner Beschäftigtenzahl einen Schwellenwert, so treten die Regelungen in Kraft und damit auch die mit ihnen verbunden Kosten. Daher wird vermutet, dass Betriebe auf ein Beschäftigungswachstum über eine Schwelle verzichten, um die damit verbunden Kosten zu vermeiden. Umgekehrt könnten Betriebe ihre Beschäftigtenzahl unter einen Schwellenwert verringern, um dadurch zusätzliche Einsparungen zu erhalten.

Wie die Analysen gezeigt haben, gehen von beschäftigtenzahlabhängigen Schwellenwerten im deutschen Arbeitsund Sozialrecht keine substanziellen Effekte auf die Arbeitsplatzdynamik in mittelständischen Firmen aus. Die Schwellenwertproblematik scheint somit weniger ausgeprägt $\mathrm{zu}$ sein als oftmals von Praktikern behauptet. Allerdings ist es für Unternehmen mit hohem personellen oder finanziellen Aufwand und Ineffizienzen verbunden, den Überblick über die sie betreffenden Schwellenwerte zu erlangen und zu behalten, weil von den rund 160 verschiedenen arbeits- und sozialrechtlichen Schwellenwerten nur wenige auf die gleiche Art berechnet werden.

Da eine Reduzierung und einheitlichere Ausgestaltung von Schwellenwerten Transaktionskosten senken und die ökonomische Effizienz erhöhen könnte, wurde bereits ein 
Reformvorschlag zur Vereinheitlichung der Berechnung von Schwellenwerten erarbeitet (vgl. Koller et al. 2007 sowie Schnabel 2007). Dieser sieht vor, dass alle Schwellenwerte einheitlich in Form von „Mindest“-Werten ausgedrückt werden und der Eingangsschwellenwert für die Anwendung von Arbeits- und sozialrechtlichen Regelungen auf ,mehr als 20 Arbeitnehmer" festgesetzt wird. Sollte eine solche einheitliche Regelung auf relativ hohem Niveau politisch nicht durchsetzbar sein, kann ersatzweise die Schwelle von ,mehr als zehn Arbeitnehmern" Verwendung finden. In Kraft treten sollen die Regelungen erst, wenn der Schwellenwert an drei aufeinanderfolgenden Stichtagen, die jeweils in der Mitte eines Quartals liegen, überschritten wird. Bezugspunkt soll in der Regel der Betrieb sein, da dieser üblicherweise die arbeitsorganisatorische Einheit für rechtliche Regelungen zum Schutz des Arbeitnehmers in seiner konkreten Arbeitssituation darstellt. Teilzeitbeschäftigte sollen einheitlich in zwei Stufen (Gewichtung der Teilzeitbeschäftigten mit weniger als 20 Stunden pro Woche mit einem Faktor von 0,5 und Teilzeitbeschäftigte mit 20 bis unter 30 Stunden pro Woche mit einem Faktor von 0,75) gewichtet werden. Dies verhindert eine überproportionale Belastung von Betrieben mit vielen Teilzeitbeschäftigten und wirkt der Aufspaltung von Arbeitsplätzen entgegen. Auszubildende, Leiharbeitnehmer und Arbeitnehmer in ruhenden Beschäftigungsverhältnissen sollen bei der Zahl der Arbeitnehmer nicht berücksichtigt werden.

Zwar wäre eine derartige Vereinheitlichung und Vereinfachung der Schwellenwertregelungen mit einer Vielzahl von Gesetzesänderungen verbunden und die Kosten für die Unternehmen würden zunächst steigen, da sie sich mit den neuen Regelungen vertraut machen müssen. In Zukunft dürfte jedoch die Überschaubarkeit erhöht und Informations- und Bürokratiekosten könnten für die Unternehmen gesenkt werden. Schwellenwerte hätten somit wieder ihre eigentliche Funktion und könnten kleine und mittlere Unternehmen vor übermäßigen Belastungen durch das Arbeits- und Sozialrecht befreien.

\section{Literatur}

Bauer, T., Bender, S., Bonin, H.: Dismissal protection and worker flows in small establishments. Economica 74, 804-821 (2007)

Burgert, D.: The impact of German job protection legislation on job creation in small establishments. Appl. Econ. Q. 52, 123-139 (2006)

Deutscher Industrie- und Handelstag, Impulse für den Arbeitsmarkt - Beschäftigungswirkungen arbeitsmarktrelevanter Gesetzeswirkungen: Ergebnisse einer DIHT-Umfrage im Frühsommer 1998 (1998)

Friedrich, W., Hägele, H.: Ökonomische Konsequenzen von Schwellenwerten im Arbeits- und Sozialrecht sowie die Auswirkungen dieser Regelungen, Studien der ISG Sozialforschung und Gesellschaftspolitik 20, Köln (1997)
Fritsch, A., Schank, T.: Betrieblicher Einsatz befristeter Beschäftigung. Soz. Fortschr. 54, 211-220 (2005)

Hagen, T., Bookmann, B.: Determinanten der Nachfrage nach befristeten Verträgen, Leiharbeit und freier Mitarbeit: Empirische Analysen auf Basis des IAB-Betriebspanels. In: Bellmann, L., Kölling, A. (Hrsg.) Betrieblicher Wandel und Fachkräftebedarf. Bd. 257, S. 199-231. Institut für Arbeitsmarkt- und Berufsforschung, Nürnberg (2002)

Institut der deutschen Wirtschaft Köln, Kündigungsschutz: Nur kosmetische Korrekturen, iwd - Informationsdienst des Instituts der deutschen Wirtschaft Köln (17), 2 (2003)

Koller, L.: Schwellenwerte im Arbeitsrecht - Regelungen an der Schwelle zur Unüberschaubarkeit. In: Merz, J. (Hrsg.) Fortschritte der MittelstandsForschung, Entrepreneurship, Professions, Small Business Economics, Bd.3, Hamburg, S. 281-305. (2007)

Koller, L.: Ökonomische Auswirkungen arbeits- und sozialrechtlicher Schwellenwerte. Peter Lang, Frankfurt (2010)

Koller, L., Schnabel, C., Wagner, J.: Arbeitsrechtliche Schwellenwerte und betriebliche Arbeitsplatzdynamik: Eine empirische Untersuchung am Beispiel des Schwerbehindertengesetzes. Z. ArbeitsmarktForsch. 39, 181-199 (2006)

Koller, L., Schnabel, C., Wagner, J.: Schwellenwerte im Arbeitsrecht: Höhere Transparenz und Effizienz durch Vereinheitlichung. Perspekt. Wirtsch.polit. 8, 242-255 (2007)

Koller, L., Schnabel, C., Wagner, J.: Freistellung von Betriebsräten - Eine Beschäftigungsbremse? Z. ArbeitsmarktForsch. 41, 305326 (2008)

Schnabel, C.: Schwellenwerte im Arbeitsrecht vereinheitlichen. Wirtschaftsdienst 87, 630-631 (2007)

Verick, S.: Threshold effects of dismissal protection legislation in Germany, IZA discussion paper 991 (2004)

Wagner, J., Schnabel, C., Kölling, A.: Wirken Schwellenwerte im deutschen Arbeitsrecht als Bremse für die Arbeitsplatzschaffung in Kleinbetrieben? In: Ehrig D., Kalmbach P. (Hrsg.) Weniger Arbeitslose - aber wie? Gegen Dogmen in der Arbeitsmarkt- und Beschäftigungspolitik, Marburg, S. 177-198 (2001)

Lena Koller studied social sciences from 1997 to 2003 at the Economics and Social Sciences Faculty of the University of ErlangenNuremberg completing her studies as Dipl.-Sozialwirtin. She acted as a researcher at IAB between 2004 and 2006 and at the University of Erlangen-Nuremberg between 2004 and 2009, gaining her doctorate there in 2009 (Dr. rer. pol.). Dr. Koller has been a senior researcher at IAB since October 2009.

Claus Schnabel studied economics at the University of Kent at Canterbury (M.A. in Economics 1985) and at Hohenheim University (Ph.D. 1988) and finished his habilitation at Ruhr University Bochum in 1997. From 1988 to 2000 he worked as researcher and finally as head of the industrial relations section at the Cologne Institute for Business Research (IW Köln). Since 2000 he has been Professor for Labour and Regional Economics at the Friedrich-Alexander University ErlangenNuremberg. His main research interests are in the fields of industrial relations, wages, collective bargaining, unions and works councils.

Joachim Wagner Study of economics at Hannover University; Diploma (1979), Dr. rer. pol. (1984) and Habilitation (1990) in Economics at Hannover University. Since 1993 Professor of Economics/Quantitative Economic Research at Leuphana University Lueneburg. Main areas of research: International firm activities, applied microeconometrics, firm level data. 